\title{
Flavor violation in meson decays
}

\author{
J. I. Aranda ${ }^{a}$, E. Cruz-Albaro ${ }^{\star a}$, D. Espinosa-Gómez ${ }^{\dagger a}$, J. Montaño $^{a, b}, \mathbf{F}$. \\ Ramírez-Zavaleta $^{a}$, E. S. Tututi ${ }^{a}$ \\ ${ }^{a}$ Facultad de Ciencias Físico Matemáticas, Universidad Michoacana de San Nicolás de \\ Hidalgo, Avenida Francisco J. Mújica S/N, 58060, Morelia, Michoacán, México \\ ${ }^{b}$ CONACYT, México \\ E-mail: feramirez@umich.mx
}

Some extended models predict the existence of a new neutral massive gauge boson, identified as the $Z^{\prime}$ boson, together with flavor-changing neutral currents. In this theoretical framework, we estimate the intensity of couplings regarding the interaction between the $Z^{\prime}$ boson with the bottom and the strange quarks through the $B_{s}^{0} \rightarrow \mu^{+} \mu^{-}$transition, which allow us to study the $B_{s}^{0} \rightarrow$ $\tau \mu, \tau e, \mu e$ decays. We present preliminary results, where the corresponding branching ratios are estimated; our predictions are contrasted with similar ones coming from several extended models. In particular, our estimates for the branching ratios range between $10^{-9}$ and $10^{-6}$.

7th Annual Conference on Large Hadron Collider Physics - LHCP2019

20-25 May, 2019

Puebla, Mexico

* Speaker.

† Speaker. 


\section{The model}

Many extensions of the Standard Model (SM) predict the existence of an extra $U^{\prime}(1)$ gauge symmetry group and its associated $Z^{\prime}$ boson, which has been an object of extensive phenomenological studies [1]. In particular, the $S U_{C}(3) \times S U_{L}(2) \times U_{Y}(1) \times U^{\prime}(1)$ extended electroweak gauge group is the simplest extended model that predicts an extra neutral gauge boson, known as $Z^{\prime}$ boson. This boson can induce flavor-changing neutral currents (FCNC) at the tree level through the $Z^{\prime} f_{i} f_{j}$ couplings, where $f_{i}$ and $f_{j}$ are always fermions of different flavor. We consider the more general renormalizable Lagrangian that includes FCNC, mediated by this new massive neutral gauge boson, which is predicted in several extended models [2, 3]:

$$
\mathscr{L}_{N C}=\sum_{i, j}\left[\bar{f}_{i} \gamma^{\alpha}\left(\Omega_{L f i f j} P_{L}+\Omega_{R f i f j} P_{R}\right) f_{j}+\bar{f}_{j} \gamma^{\alpha}\left(\Omega_{L f j f i}^{*} P_{L}+\Omega_{R f j f i}^{*} P_{R}\right) f_{i}\right] Z_{\alpha}^{\prime},
$$

where $P_{L, R}$ are the chiral projectors and $Z_{\alpha}^{\prime}$ represents the new neutral massive gauge boson. The $\Omega_{L f_{i} f_{j}}, \Omega_{R f_{i} f_{j}}$ parameters represent the strength of the $Z^{\prime} f_{i} f_{j}$ couplings. For simplicity, we assume that $\Omega_{L f_{i} f_{j}}=\Omega_{L f_{j} f_{i}}$ and $\Omega_{R f_{i} f_{j}}=\Omega_{R f_{j} f_{i}}$. The Lagrangian in Eq. (1.1) contains both flavorconserving and flavor-violating couplings. The flavor-conserving couplings, $Q_{L, R}^{f_{i}}$ [4], are related to the $\Omega$ couplings as $\Omega_{L f_{i} f_{i}}=-g_{2} Q_{L}^{f_{i}}$ and $\Omega_{R f_{i} f_{i}}=-g_{2} Q_{R}^{f_{i}}$, where $g_{2}$ is the gauge coupling of the $Z^{\prime}$ boson. Here, we only consider the following $Z^{\prime}$ bosons: the $Z_{S}$ of the sequential $Z$ model, the $Z_{L, R}$ of the left-right symmetric model, the $Z_{\chi}$ arising from the breaking of $S O(10) \rightarrow S U(5) \times U(1)$, the $Z_{\psi}$ resulting from $E_{6} \rightarrow S O(10) \times U(1)$, and the $Z_{\eta}$ arising in many superstring-inspired models. The different models are distinguished by their gauge coupling with the $Z^{\prime} s$ boson

$$
g_{2}=\sqrt{5 / 3} \sin \theta_{W} g_{1} \lambda_{g},
$$

where $g_{1}=g / \cos \theta_{W}$ and $\lambda_{g}$ is a parameter that depends of the symmetry breaking pattern, which is commonly assumed $\mathscr{O}(1)$ [5]. In the sequential $Z_{S}$ model, the gauge coupling $g_{2}=g_{1}$.

\section{The decay}

The effective Hamiltonian that describes the $B_{s}^{0} \rightarrow l_{i} l_{j}$ process (see Fig. 1(a)) can be expressed as follows [6]

$$
\begin{aligned}
\mathscr{H}_{e f f}= & \frac{C_{\mathrm{eff}}\left(m_{b}\right)}{m_{B_{s}^{0}}^{2}-m_{Z^{\prime}}^{2}+i m_{Z^{\prime}} \Gamma_{Z^{\prime}}}\left\{\left[\bar{s}\left(p_{2}\right) \gamma^{\mu}\left(\Omega_{L b s} P_{L}+\Omega_{R b s} P_{R}\right) b\left(p_{1}\right)\right]\right. \\
& \times\left[\overline{l_{i}}\left(p_{3}\right) \gamma^{\mu}\left(\Omega_{L l_{i} l_{j}} P_{L}+\Omega_{R l_{i} l_{j}} P_{R}\right) l_{j}\left(p_{4}\right)\right]+\left[\overline { s } ( p _ { 2 } ) \gamma ^ { \mu } \left(\Omega_{L b s} P_{L}\right.\right. \\
& \left.\left.\left.+\Omega_{R b s} P_{R}\right) b\left(p_{1}\right)\right]\left[\overline{l_{j}}\left(p_{4}\right) \gamma^{\mu}\left(\Omega_{L l_{j} l_{i}}^{*} P_{L}+\Omega_{R l_{j} l_{i}}^{*} P_{R}\right) l_{i}\left(p_{3}\right)\right]\right\},
\end{aligned}
$$

where $\Gamma_{Z^{\prime}}$ is the total decay width of the $Z^{\prime}$ boson, $m_{B_{s}^{0}}$ is the $B_{s}^{0}$ meson mass, and $C_{\text {eff }}\left(m_{b}\right)$ is the respective Wilson coefficient. To calculate the transition amplitude $\left\langle 0\left|\mathscr{H}_{\text {eff }}\right| B_{s}^{0}\right\rangle$, one can generally adopt the vacuum insertion method for the evaluation of the matrix elements in Eq. (2.1), which are given in general as

$$
\left\langle 0\left|\bar{s} \gamma^{\mu} \gamma_{5} b\right| B_{s}^{0}\right\rangle=i f_{B_{s}^{0}} P^{\mu},\left\langle 0\left|\bar{q} \gamma^{\mu} b\right| B_{q}^{0}\right\rangle=0,
$$

where $P$ is the momentum of $B_{s}^{0}$ meson. 


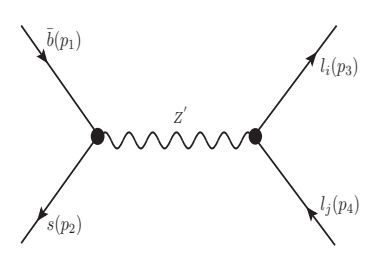

(a)
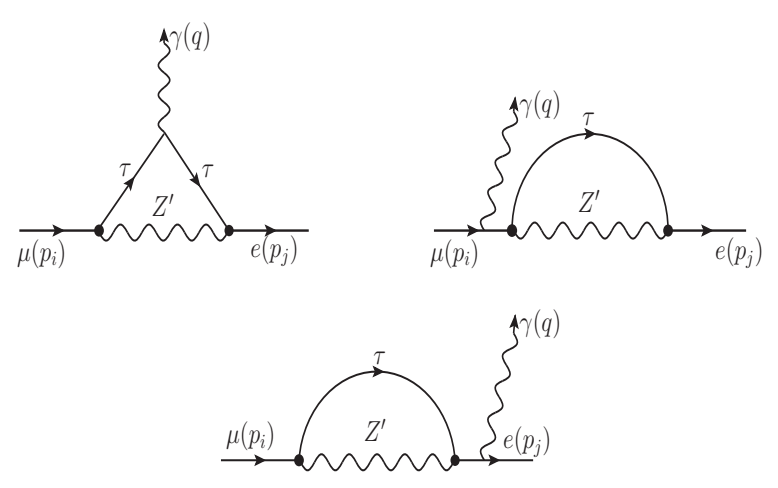

(b)

Figure 1: Feynman diagrams that represent the decays: a) $B_{s}^{0} \rightarrow l_{i} l_{j}$ and b) $\mu \rightarrow e \gamma$. Both processes are mediated by a $Z^{\prime}$ gauge boson.

By using Eq. (2.2) and assuming that $\Omega_{R b s}-\Omega_{L b s} \equiv \Omega_{b s}$, the amplitudes for the $B_{s}^{0} \rightarrow l_{i} l_{j}$ decays can be written as

$$
\begin{aligned}
\mathscr{M}\left(B_{s}^{0} \rightarrow \bar{l}_{i} l_{j}\right)= & \frac{i}{2} \frac{f_{B_{s}^{0}} C_{\mathrm{eff}}\left(m_{b}\right) \Omega_{b s}}{m_{B_{s}^{0}}^{2}-m_{Z^{\prime}}^{2}+i m_{Z^{\prime}} \Gamma_{Z^{\prime}}} \bar{l}_{i}\left(p_{4}\right)\left[\left(m_{l_{i}} \Omega_{R l_{i} l_{j}}-m_{l_{j}} \Omega_{L l_{i} l_{j}}\right) P_{R}\right. \\
+ & \left.\left(m_{l_{i}} \Omega_{L l_{i} l_{j}}-m_{l_{j}} \Omega_{R l_{i} l_{j}}\right) P_{L}\right] l_{j}\left(p_{3}\right), \\
\mathscr{M}\left(B_{s}^{0} \rightarrow l_{i} \bar{l}_{j}\right)= & \frac{i}{2} \frac{f_{B_{s}^{0}} C_{\mathrm{eff}}\left(m_{b}\right) \Omega_{b s}}{m_{B_{s}^{0}}^{2}-m_{Z^{\prime}}^{2}+i m_{Z^{\prime}} \Gamma_{Z^{\prime}}} \bar{l}_{j}\left(p_{3}\right)\left[\left(m_{l_{j}} \Omega_{R l_{i} l_{j}}^{*}\right.\right. \\
& \left.\left.-m_{l_{i}} \Omega_{L l_{j} l_{i}}^{*}\right) P_{R}+\left(m_{l_{j}} \Omega_{L l_{i} l_{j}}^{*}-m_{l_{i}} \Omega_{R l_{j} l_{i}}^{*}\right) P_{L}\right] l_{i}\left(p_{4}\right) .
\end{aligned}
$$

The decay width of the $B_{s}^{0} \rightarrow l_{i} l_{j}$ process is

$$
\begin{aligned}
\Gamma\left(B_{s}^{0}\right. & \left.\rightarrow l_{i} l_{j}\right)=\frac{C_{\mathrm{eff}}^{2}\left(m_{b}\right)\left|\Omega_{b s}\right|^{2} m_{B_{s}^{0}}^{3} f_{B_{s}^{0}}^{2}}{32 \pi\left[\left(m_{B_{s}^{0}}^{2}-m_{Z^{\prime}}^{2}\right)^{2}+m_{Z^{\prime}}^{2} \Gamma_{Z^{\prime}}^{2}\right]}\left\{\left(\left|\Omega_{L_{l} l_{j}}\right|^{2}+\left|\Omega_{R l_{i} l_{j}}\right|^{2}\right)\right. \\
& \left.\times\left[\frac{\left(m_{l i}^{2}+m_{l j}^{2}\right)}{m_{B_{s}^{0}}^{2}}-\frac{\left(m_{l i}^{2}-m_{l j}^{2}\right)^{2}}{m_{B_{s}^{0}}^{4}}\right]-\frac{4 m_{l i} m_{l j}}{m_{B_{s}^{0}}^{2}} \operatorname{Re}\left(\Omega_{R l_{i} l_{j}} \Omega_{R_{i} l_{j} l_{j}}^{*}\right)\right\} \\
& \times \sqrt{\left[1-\frac{\left(m_{l_{j}}+m_{l_{i}}\right)^{2}}{m_{B_{s}^{0}}^{2}}\right]\left[1-\frac{\left(m_{l_{i}}-m_{l_{j}}\right)^{2}}{m_{B_{s}^{0}}^{2}}\right]} .
\end{aligned}
$$

In the following, we suppose that $\Omega_{L l_{i} l_{j}}=\Omega_{R l_{i} l_{j}}=\Omega_{l_{i} l_{j}}$.

In accordance with experimental conditions we need to account for the sizable effect of the $B_{s}^{0}-\overline{B_{s}^{0}}$ mixing, in which the decay width difference between the $B_{s}^{0}$-mass eigenstates is crucial [7]. In this sense,

$$
\operatorname{Br}\left(B_{s}^{0} \rightarrow l_{i} l_{j}\right)=\tau_{B_{s}^{0}} \Gamma\left(B_{s}^{0} \rightarrow l_{i} l_{j}\right) \simeq\left(1-y_{s}\right) \operatorname{Br}\left(B_{s}^{0} \rightarrow l_{i} l_{j}\right)_{\operatorname{Exp}}
$$

where $\tau_{B_{s}^{0}}$ is the mean life of the $B_{s}^{0}$ meson, $y_{s}=\Delta \Gamma_{B_{s}^{0}} /\left(2 \Gamma_{B_{s}^{0}}\right)$ is the correction factor, being $\Gamma_{B_{s}^{0}}$ the average decay width of $B_{s}^{0}$ and $\Delta \Gamma_{B_{s}^{0}}$ stands for the width difference between the $B_{s}^{0}$-mass eigenstates. 


\section{Estimation of the $Z^{\prime} b s$ coupling from the $B_{s}^{0} \rightarrow \mu^{+} \mu^{-}$decay}

In the following, we are going to derive the expression for the $\Omega_{b s}$, which represents the intensity of th $Z^{\prime} b s$ coupling, by using the $B_{s}^{0} \rightarrow \mu^{+} \mu^{-}$process, to this purpose, it is resorted to Eq. (2.5). Since the $B_{s}^{0} \rightarrow \mu^{+} \mu^{-}$decay was already measured [8], we will assume that within the experimental uncertainty the new physics effects could be found. Thereby,

$$
\Delta \Gamma\left(B_{s}^{0} \rightarrow \mu \bar{\mu}\right)_{\operatorname{Exp}}=\frac{g_{2}^{2} C_{e f f}^{2}\left(m_{b}\right)\left|\Omega_{b s}\right|^{2} m_{B_{s}^{0}} f_{B_{s}^{0}}^{2} m_{\mu}^{2}}{32 \pi\left[\left(m_{B_{s}^{0}}^{2}-m_{Z^{\prime}}^{2}\right)^{2}+m_{Z^{\prime}}^{2} \Gamma_{Z^{\prime}}^{2}\right]}\left|Q_{L}^{\mu}-Q_{R}^{\mu}\right|^{2} \sqrt{1-\frac{4 m_{\mu}^{2}}{m_{B_{s}^{0}}^{2}}},
$$

where $\Omega_{L, R_{\mu \mu}}=-g_{2} Q_{L, R}^{\mu}$. Finally, when inserting Eq. (3.1) into Eq. (2.6) we obtain

$$
\left|\Omega_{b s}\right|^{2}=\frac{32 \pi\left(1-y_{s}\right)\left[\left(m_{B_{s}^{0}}^{2}-m_{Z^{\prime}}^{2}\right)^{2}+m_{Z^{\prime}}^{2} \Gamma_{Z^{\prime}}^{2}\right] \Delta \operatorname{Br}\left(B_{s}^{0} \rightarrow \mu \bar{\mu}\right)_{\operatorname{Exp}}}{\tau_{B_{s}^{0}} g_{2}^{2} C_{\mathrm{eff}}^{2}\left(m_{b}\right) m_{B_{s}^{0}} f_{B_{s}^{0}}^{2} m_{\mu}^{2}\left|Q_{L}^{\mu}-Q_{R}^{\mu}\right|^{2} \sqrt{1-\frac{4 m_{\mu}^{2}}{m_{B_{s}^{0}}^{2}}}} .
$$

It should be recalled that the last equation represents a bound over the strength of the $Z^{\prime} b s$ coupling.

\section{Constraining the $Z^{\prime} \mu e$ coupling from $\mu-e$ conversion}

We will estimate the $\Omega_{\mu e}$ parameter through the $\mu \rightarrow e \gamma$ decay resorting to the $\mu-e$ conversion, where the contributions of the flavor-violating vertex, $Z^{\prime} \mu e$, to the $\mu \rightarrow e \gamma$ process are given by the Feynman diagrams shown in Fig. 1(b). Therefore, we can write the associated branching ratio as follows

$$
B r(\mu \rightarrow e \gamma)=\frac{\alpha}{2}\left(1-x^{2}\right)^{3}\left[\left|\Omega_{\mu \tau} \Omega_{e \tau}\right|^{2}\left|y_{1}+y_{2}+y_{3}+y_{4}\right|^{2}\right] \frac{m_{\mu}}{\Gamma_{\mu}},
$$

where $x=\frac{m_{e}}{m_{\mu}}$ and $\Gamma_{\mu}$ is the total decay width of the muon. The $y_{1}, y_{2}, y_{3}$ and $y_{4}$ variables contain the loop contributions and are explicitly given in Ref. [9]. The $\Omega_{\mu e}$ parameter can be extracted by using Eq. (4.1) along with the conversion rate in titanium nuclei, $C R(\mu T i \rightarrow e T i) \cong \frac{1}{200} \operatorname{Br}(\mu \rightarrow e \gamma)$ [10]. In order to bound $\Omega_{\mu e}$, we propose two scenarios:

(a) First case: By supposing that $\Omega_{\mu \tau} \Omega_{e \tau}=\Omega_{\mu e}$, it is found that $\left|\Omega_{\mu e}\right|^{2}$ can be expressed

$$
\left|\Omega_{\mu e}\right|^{2}<400 \frac{\Gamma_{\mu}}{m_{\mu}} \frac{C R(\mu T i \rightarrow e T i)}{\alpha\left(1-x^{2}\right)^{3}\left|y_{1}+y_{2}+y_{3}+y_{4}\right|^{2}} .
$$

(b) Second case: By considering that $\Omega_{\mu \tau} \Omega_{e \tau}=\Omega_{\tau \tau} \Omega_{\mu e}$, it is found that

$$
\left|\Omega_{\mu e}\right|^{2}<400 \frac{\Gamma_{\mu}}{m_{\mu}} \frac{C R(\mu T i \rightarrow e T i)}{\alpha\left(1-x^{2}\right)^{3}\left|\Omega_{\tau \tau}\right|^{2}\left|y_{1}+y_{2}+y_{3}+y_{4}\right|^{2}} .
$$

The former scenarios can be justified by thinking that there is an effective coupling between four charged leptons $\mu e \tau \tau$, for example, through a dispersion $\mu e \rightarrow \tau \tau$ mediated by a $Z^{\prime}$ gauge boson. 


\section{Results and conclusions}

In order to estimate values for the $\Omega_{b s}$ parameter and branching ratios for the $B_{s}^{0} \rightarrow \tau \mu, \tau e, \mu e$ processes, we use the following input data: $m_{\mu}=0.105 \mathrm{GeV}, m_{e}=0.00051099 \mathrm{GeV}, m_{\tau}=1.77686$ $\mathrm{GeV}, m_{B_{s}^{0}}=5.3668 \mathrm{GeV}, f_{B_{s}^{0}}=0.230 \mathrm{GeV}, \tau_{B_{s}^{0}}=2.2876 \times 10^{12} \mathrm{GeV}^{-1}, \operatorname{Br}\left(B_{s}^{0} \rightarrow \mu \bar{\mu}\right)_{\operatorname{Exp}}=(3.0 \pm$ $\left.0.6_{-0.2}^{+0.3}\right) \times 10^{-9}$ [8], $\Delta \operatorname{Br}\left(B_{s}^{0} \rightarrow \mu \bar{\mu}\right)_{\text {Exp }}=0.6 \times 10^{-9}, y_{s}=0.065$ and $C R(\mu T i \rightarrow e T i)<4.3 \times$ $10^{-12}[11,12]$. The Fig. 2(a) shows the behavior of $\left|\Omega_{b s}\right|^{2}$ as a function of the $Z^{\prime}$ boson mass for the different models considered. The mass range corresponds to the interval $m_{Z^{\prime}}=[2,6] \mathrm{TeV}$, which is in strict accordance with current experimental restrictions. From Fig. 2, it can be appreciated that the $Z_{\eta}$ boson is the responsible for the highest value, while for the same mass interval, the $Z_{\chi}$ provides the lowest one. Regarding the $B_{s}^{0} \rightarrow \tau \mu, \tau e$ decays, we estimate the $\Omega_{\tau \mu}$ and $\Omega_{\tau e}$ parameters just as in Ref. [13], by using experimental upper limits on the $\tau \rightarrow e e \bar{e}$ and $\tau \rightarrow \mu \mu \bar{\mu}$ decays [11]. In Fig. 2(b), it can be observed that the $Z_{\eta}$ boson contribution is $\operatorname{Br}\left(B_{s}^{0} \rightarrow \tau e\right) \sim 10^{-6}$, for the mass interval $m_{Z^{\prime}}=[2,3] \mathrm{TeV}$; while that for the $B_{s}^{0} \rightarrow \tau \mu$ decay (Fig. 2(c)), once again, the $Z_{\eta}$ boson offers the most intense contribution, being of the order of $10^{-6}$ for the same $m_{Z^{\prime}}$ range.

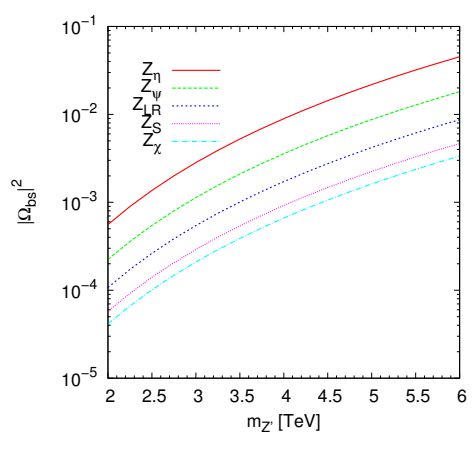

(a)

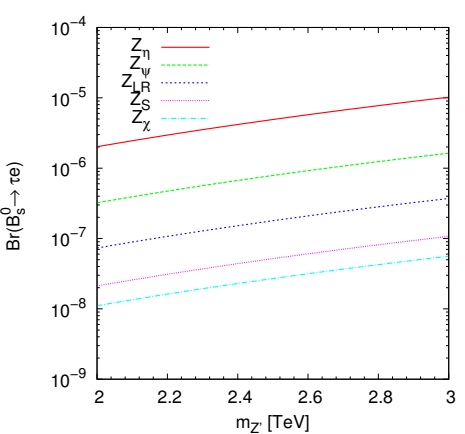

(b)

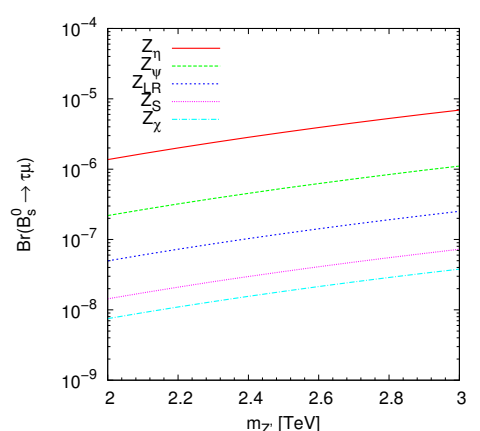

(c)

Figure 2: (a) The parameter $\left|\Omega_{b s}\right|^{2}$ as a function of the $Z^{\prime}$ boson mass. (b) $\operatorname{Br}\left(B_{s}^{0} \rightarrow \tau e\right.$ ) as a function of $m_{Z^{\prime}}$ (c) $\operatorname{Br}\left(B_{s}^{0} \rightarrow \tau \mu\right)$ as a function of $m_{Z^{\prime}}$.

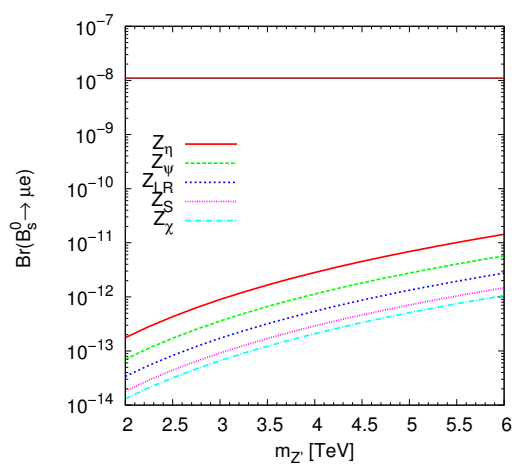

(a)

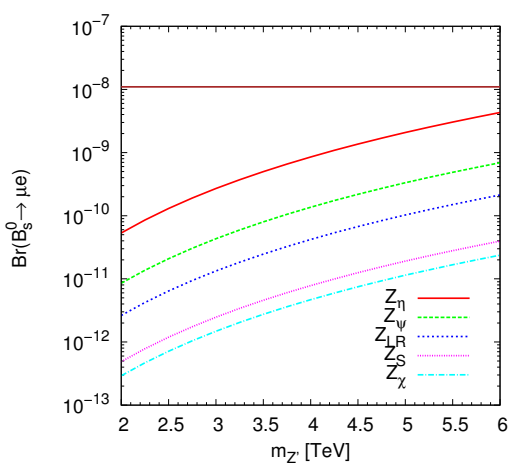

(b)

Figure 3: (a) $\operatorname{Br}\left(B_{s}^{0} \rightarrow \mu e\right)$ for the scenario $\Omega_{\mu \tau} \Omega_{\tau e}=\Omega_{\mu e}$ and (b) $\operatorname{Br}\left(B_{s}^{0} \rightarrow \mu e\right)$ for the scenario $\Omega_{\mu \tau} \Omega_{\tau e}=$ $\Omega_{\tau \tau} \Omega_{\mu e}$. The horizontal line represents the experimental bound for $\operatorname{Br}\left(B_{s}^{0} \rightarrow \mu e\right)_{\operatorname{Exp}}<1.1 \times 10^{-8}$. 
In Fig. 3 the numerical results for the $\operatorname{Br}\left(B_{s}^{0} \rightarrow \mu e\right)$ are presented. From this figure we observe that the $Z_{\eta}$ is responsible for the main contribution, while the lowest one corresponds to the $Z_{\chi}$ boson. In particular, for scenario (a), the $Z_{\eta}$ boson offers a $\operatorname{Br}\left(B_{s}^{0} \rightarrow \mu e\right) \sim 10^{-13}$ in $m_{Z^{\prime}}=[2,3]$ $\mathrm{TeV}, \operatorname{Br}\left(B_{s}^{0} \rightarrow \mu e\right) \sim 10^{-12}$ in $m_{Z^{\prime}}=[3.1,5.4] \mathrm{TeV}$ and $\mathrm{Br}\left(B_{s}^{0} \rightarrow \mu e\right) \sim 10^{-11}$ in $m_{Z^{\prime}}=[5.5,6]$ $\mathrm{TeV}$; whereas for scenario (b), $\operatorname{Br}\left(B_{s}^{0} \rightarrow \mu e\right) \sim 10^{-11}$ in $m_{Z^{\prime}}=[2,2.3] \mathrm{TeV}, \operatorname{Br}\left(B_{s}^{0} \rightarrow \mu e\right) \sim 10^{-10}$ in $m_{Z^{\prime}}=[2.4,4.1] \mathrm{TeV}$ and $\operatorname{Br}\left(B_{s}^{0} \rightarrow \mu e\right) \sim 10^{-9}$ in $m_{Z^{\prime}}=[4.2,6] \mathrm{TeV}$, being approximately one order of magnitude lower than the experimental bound [11].

\section{References}

[1] M. Cvetic, P. Langacker and B. Kayser, Phys. Rev. Lett. 68, 2871 (1992); M. Cvetic and P. Langacker, Phys. Rev. D 54, 3570 (1996).

[2] P. Langacker and M. Luo, Phys. Rev. D 45, 278 (1992).

[3] J. I. Aranda, J. Montaño, F. Ramírez-Zavaleta, J. J. Toscano, and E. S. Tututi, Phys. Rev. D 82, 054002 (2010).

[4] R. W. Robinett and J. L. Rosner, Phys. Rev. D 26, 2396 (1982); P. Langacker and M. Luo, Phys. Rev. D 45, 278 (1992).

[5] R. W. Robinett and J. L. Rosner, Phys. Rev. D 25, 3036 (1982); 27, 679(E) (1983); R. W. Robinett, Phys. Rev. D 26, 2388 (1982).

[6] M. K. Gaillard and B. W. Lee, Phys. Rev. D 10, 897 (1974); R. Mohanta, Phys. Rev. D 71, 114013 (2005); S. Sahoo, D. Banerjee, M. Kumar, and S. Mohanty, Int. J. Mod. Phys. 27, 1250184 (2012).

[7] K. De Bruyn, R. Fleischer, R. Knegjens, P. Koppenburg, M. Merk, and N. Tuning, Phys. Rev. Lett. 109, 041801 (2012); A. Lenz and U. Nierste, arXiv:1102.4274.

[8] R. Aaij et al., (LHCb Collaboration), Phys. Rev. Lett. 111, 101805 (2013); R. Aaij et al., (LHCb Collaboration), Phys. Rev. Lett. 118, 191801 (2017).

[9] L. Lavoura, Eur. Phys. J. C 29, 191 (2003).

[10] D. N. Dinh, A. Ibarra, E. Molinaro, and S. T. Petcov, JHEP 08, 125 (2012); J. Hisano and K. Tobe, Phys. Lett. B 510, 197 (2001).

[11] M. Tanabashi et al., Phys. Rev. D 98, 030001 (2018).

[12] C. Dohmen et al., (SINDRUM II Collaboration), Phys. Lett. B 317, 631 (1993).

[13] J. I. Aranda, J. Montaño, F. Ramírez-Zavaleta, J. J. Toscano, and E. S. Tututi, Phys. Rev. D 86, 035008 (2012). 